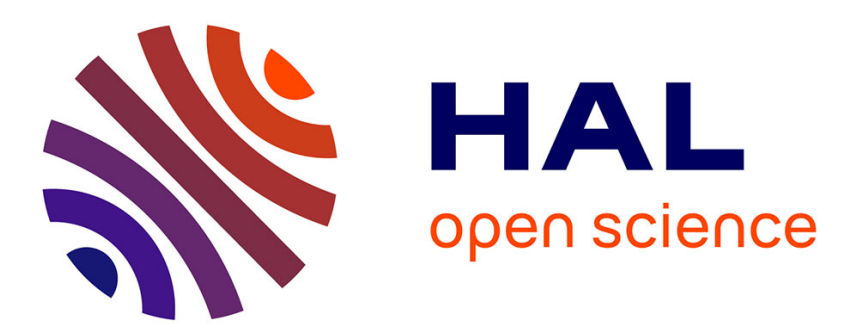

\title{
Reciprocity calibration techniques, implementation on the OpenAirInterface platform
}

\author{
Boris Kouassi, Bassem Zayen, Irfan Ghauri, Luc Deneire
}

\section{To cite this version:}

Boris Kouassi, Bassem Zayen, Irfan Ghauri, Luc Deneire. Reciprocity calibration techniques, implementation on the OpenAirInterface platform. CogART 2011, Oct 2011, Barcelone, Spain. pp.372-376, 10.1145/2093256.2093282. hal-00742987

\section{HAL Id: hal-00742987 \\ https://hal.science/hal-00742987}

Submitted on 18 Oct 2012

HAL is a multi-disciplinary open access archive for the deposit and dissemination of scientific research documents, whether they are published or not. The documents may come from teaching and research institutions in France or abroad, or from public or private research centers.
L'archive ouverte pluridisciplinaire HAL, est destinée au dépôt et à la diffusion de documents scientifiques de niveau recherche, publiés ou non, émanant des établissements d'enseignement et de recherche français ou étrangers, des laboratoires publics ou privés. 


\title{
Reciprocity Calibration Techniques, Implementation on the OpenAirInterface Platform
}

\author{
Boris Kouassi ${ }^{1}$, Bassem Zayen ${ }^{2}$, Irfan Ghauri ${ }^{3}$ and Luc Deneire ${ }^{1}$ \\ ${ }^{1}$ Laboratoire I3S, Sophia Antipolis, France \\ ${ }^{2}$ EURECOM, Sophia Antipolis, France \\ ${ }^{3}$ Intel Mobile Communications, Sophia Antipolis, France \\ Email:kouassi@i3s.unice.fr, bassem.zayen@eurecom.fr, irfan.ghauri@intel.com,deneire@i3s.unice.fr
}

\begin{abstract}
The channel reciprocity is an advantageous hypothesis in cognitive radio (CR) technologies, however it is not practically exploitable due to miscellaneous discrepancies including the radio frequency ( $\mathrm{RF}$ ) impairments. Consequently, previous studies proposed calibration solutions to solve these RF impairments. This paper is a step ahead in the implementation of calibration algorithms through the EURECOM's experimental OpenAirInterface (OAI) platform. It proposes to implement and evaluate a less computationally intensive multiple-input multiple-output (MIMO) calibration algorithm, according to a realistic scenario based on long term evolution-time division duplex (LTE-TDD) specification. The result from OAI simulator shows that it is feasible to restore the reciprocity after calibration in a non reciprocal channel, and provides an overview of the challenges in the channel estimation in a real time case.
\end{abstract}

\section{Categories and Subject Descriptors}

C.2 [Computer-Communication Networks]: Miscellaneous; C.4 [Performance of Systems]: Performance attributes; D.4.7 [Real-time systems and embedded systems]:

\section{General Terms}

Algorithms, Performance

\section{Keywords}

Cognitive radio, reciprocity calibration, MIMO/LTE-TDD, beamforming, channel estimate.

\section{INTRODUCTION}

In cognitive radio (CR) systems, the radio transmission is enhanced by combining all the available information in the network [1] [2]. A proposal to achieve this goal, denoted

Permission to make digital or hard copies of all or part of this work for personal or classroom use is granted without fee provided that copies are not made or distributed for profit or commercial advantage and that copies bear this notice and the full citation on the first page. To copy otherwise, to republish, to post on servers or to redistribute to lists, requires prior specific permission and/or a fee.

CogART'11, October 26-29, Barcelona, Spain

Copyright (C) 2011 ACM ISBN 978-1-4503-0912-7/11/10 ...\$10.00. interweave CR suggests to exploit all the available spatiotemporal holes in the radio spectrum [3].

This study highlights several methods drawn on interweave $\mathrm{CR}$ techniques to promote the coexistence between a primary licensed system and a secondary system [3]. We focus especially on beamforming (BF) and calibration techniques. Specifically, a persistent problem in CR is to find the best ways for secondary users to transmit in a licensed radio environment without disturbing the primary users. In this respect, the $\mathrm{BF}$ is an interesting technique, since it uses an antennas array to steer a signal towards a specific receiver, then maximizes the signal to noisy ratio (SNR) for the considered receiver [4]. Otherwise, till now, feedback techniques are commonly used to perform $\mathrm{BF}$ at the transmitter, but a feedback procedure is impossible between the primary receiver and the secondary transmitter. Therefore, in [5], a BF solution was proposed which contrasts with the feedback approach. It consists in using the reciprocity of the electromagnetic channel and trying to reduce channel estimation and feedback overhead.

But in a real-time transmission, the reciprocity is usable only after a calibration procedure, because of several perturbations, including the discrepancy between radio frequency circuits in a multiple-input multiple-output (MIMO) system. Thus, from the study presented in [5], we selected a less computationally intensive multi-input multi-output (MIMO) calibration method consisting in dividing the MIMO channel into a single channel, then the calibration is finally performed for each subdividing channel, by total leastsquares (TLS) techniques $[6,7,8]$.

The main objective in this paper is to embed the selected MIMO calibration algorithm in an experimental platform. To this end, we introduced a real time scenario including two secondary users in a system based on long term evolutiontime division duplex (LTE-TDD) specification [9]. Finally, the practical involvements of the MIMO calibration algorithm implementation are assessed using EURECOM's OpenAir Interface (OAI) platform, specifically the OAI simulation part $[10,11]$. The OAI platform consists of a simulation, emulation and real time experiment platform which tests the validity of concepts in radio networks.

This study reveals the results, limits and the perspectives represented by the specified MIMO calibration approach. It also participates to the CR concept demonstration of the European consortium project CROWN (cognitive radio oriented wireless networks). This project, involving a set of universities and companies, aims at understanding and 
analysing methods related to CR [12].

The rest of this paper is structured as follows. The Section 2 shows the system model, and describes the basis elements of our study. In Section 3 we detail the essential of the experimental OAI platform then the result from OAI simulator are illustrated in Section 4. Finally, the conclusions and prospects are depicted in Section 5.

\section{SYSTEM MODEL}

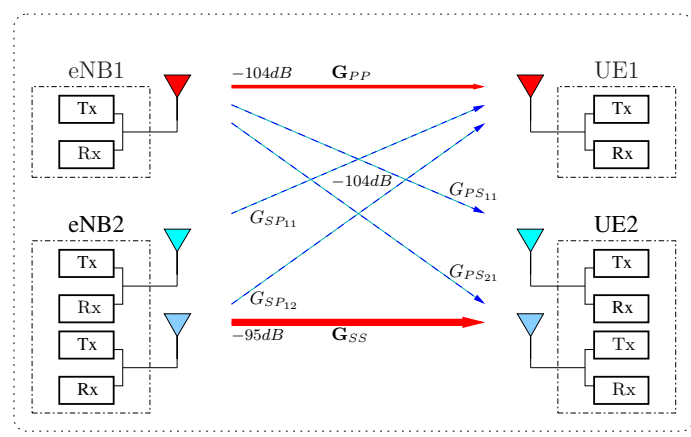

Figure 1: Illustration of primary system including eNB1 and UE1, and secondary system, eNB2 and UE2, with the corresponding path attenuation for each channels.

Fig. 1 presents the transmission model consisting of a primary system including the licensed users, eNB1 and UE1 designed with one antenna, and a secondary system composed of opportunistic users, eNB2 and UE2 with two antennas, which are not licensed to transmit in the radio environment. The denomination eNodeB (eNB) and User Equipment (UE) is conform to LTE, and depicts a communication including one node and an attached LTE equipment. Orthogonal frequency division multiplexing (OFDM) is the modulation of choice for the proposed scenario over a time division duplex (TDD) mode. The radio frequency circuitry (RF) is represented on Fig. 1 by the transmission and reception filters Tx and Rx for each antenna. In the proposed model, we take into account the path loss in free space:

$$
P_{l s}=20 \log _{10}\left(\frac{4 \pi d}{\lambda}\right)
$$

where $d$ is the distance between the transmit and receive antennas, and $\lambda$ the wavelength. The predetermined primary system is defined with a pathloss equal to $-104 d B$, and the secondary users are supposed to be closer, implying a secondary link pathloss equal to $-95 d B$.

Considering that primary and secondary systems share the same spectrum, the objective in this work is to exploit interweave $\mathrm{CR}$ techniques (including $\mathrm{BF}$ and synchronization) in order to allow secondary transmission, while avoiding to disturb the primary licensed communication. However, due to the impossibility for the secondary system to communicate with the primary one, we propose to exploit channel reciprocity in TDD to determine the channel state information (CSI) [5, 13].

In [13], a technique to avoid crosslink calibration was presented. This technique consists in designing the beamformer subspace in MIMO interference channel with a TDD transmission scheme. The authors in [13] do not require a priori knowledge of the crosslink CSI at the secondary transmitters. They are able to obtain information required for $\mathrm{Tx}$ beamforming through smart exploitation of received signal during a TDD time slot. We will adapt in this paper the same framework given in [13], nevertheless, we suppose here that the required downlink crosslink channel (from eNB2 to UE1) estimations are known to the secondary transmitter. In the proposed solution, the calibration parameters at the secondary transmitters allow to estimate the crosslink channel without a feedback from the primary system, as can be shown in Fig. 2.

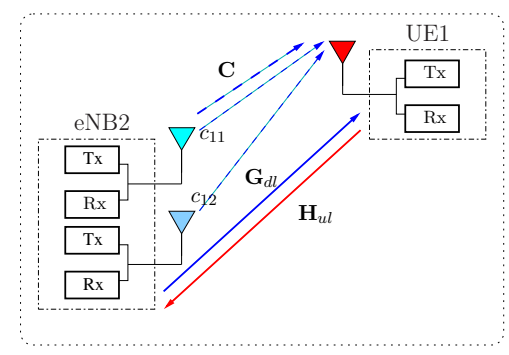

Figure 2: Description of crosslink channel between secondary eNB and the primary $\mathrm{UE}$, it is normally possible for secondary eNB to estimate the uplink crosslink channel (in listening the primary pilots) but not the downlink

The proposed interference avoidance technique is a simple zero forcing beamforming ( $\mathrm{ZF}-\mathrm{BF}$ ) technique where the secondary transmitter sends a null in the direction of the primary receiver:

$$
\mathbf{v G}_{d l} \approx 0
$$

where $\mathbf{v}$ is the BF vector at the secondary transmitter. To achieve this process, the vector $\mathbf{v}$ is composed of the reverse channel estimated through calibration, thus cancelling the interference to the primary caused by the secondary transmission.

In [5], the theoretical performance of three calibration techniques are described, subsequently, this paper goes further in addressing the implementation of the first less computational technique. Briefly, it simplifies the MIMO calibration problem in subdividing the MIMO channel into several single channels and then uses a Total Least Square (TLS) technique to solve the calibration [7] [5].

More concretely the algorithm restores the reciprocity assumption by finding the calibration parameters $\mathbf{P}$ consisting of RF filters (Tx, Rx in Fig. 2).

$$
\begin{gathered}
\mathbf{G}_{d l}=\mathbf{P}_{U E 1} \cdot \mathbf{H}_{u l}^{T} \cdot \mathbf{P}_{e N B 2} \\
\mathbf{G}_{d l}=\mathbf{R}_{U E 1} \cdot \mathbf{C} \cdot \mathbf{T}_{e N B 2}, \mathbf{H}_{u l}=\mathbf{R}_{e N B 2} \cdot \mathbf{C}^{T} \cdot \mathbf{T}_{U E 1}
\end{gathered}
$$

$\mathbf{P}_{e N B 2}=\mathbf{R}_{e N B 2}^{-T} \cdot \mathbf{T}_{e N B 2}, \mathbf{P}_{U E 1}=\mathbf{R}_{U E 1} \cdot \mathbf{T}_{U E 1}^{-T}$, and $\mathbf{C}$ is the channel matrix between antennas.

Furthermore, we aim to investigate the variation of $\mathrm{RF}$ distortions, since it directly affects the stability of calibration parameters $\mathbf{P}$ (according to temperature, variation of the environment near the antennas, ...).

The next section describes the experimental OpenAirInterface platform used to achieve the reciprocity calibration procedure. 


\section{OPENAIRINTERFACE PLATFORM}

Our objective within this work is to implement the selected calibration algorithm on the OAI platform to assess its performance in a practical case. This platform consists of several development tools composed of telecommunication hardware and real-time (free) software. Moreover, it explores the possible enhancement in the signal processing and radio transmission field $(802.16 \mathrm{~m}$ and 3GPP LTE standards), in terms of algorithms reliability, spectral and protocol efficiency, just to mention a few [10]. It allows to experiment and possibly implement new concepts in radio networks, through three main parts denoted the simulator, the emulator, and the real time experiment equipments [10].

In the context of CROWN project [12], the scenario given in Fig. 1 will be implemented on EURECOM's OAI platform for real time experiments. In the proposed scenario, one antenna will be considered for primary transmitter and receiver. On the other side, two antennas will be considered for secondary users. CBMIMO1 cards will be used to transmit and receive signals, these cards as OAI antennas were given in Fig. 3. However, this paper focuses on the simulation part of OAI which represents a first step for a real time implementation in the LTE compliant OAI hardware interface.

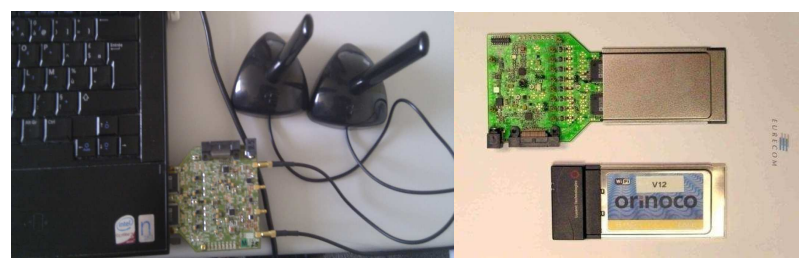

Figure 3: Real time architecture with CBMIMO card and antennas. (I3S \& EURECOM Pictures).

Moreover, our work in OAI assumes the $10 \mathrm{~ms}$ periodic TDD frame structure type 2 , with the configuration number 3 as described in Fig. 4. It is composed of 20 subframes with the OFDM multiplexing in the uplink and the downlink.

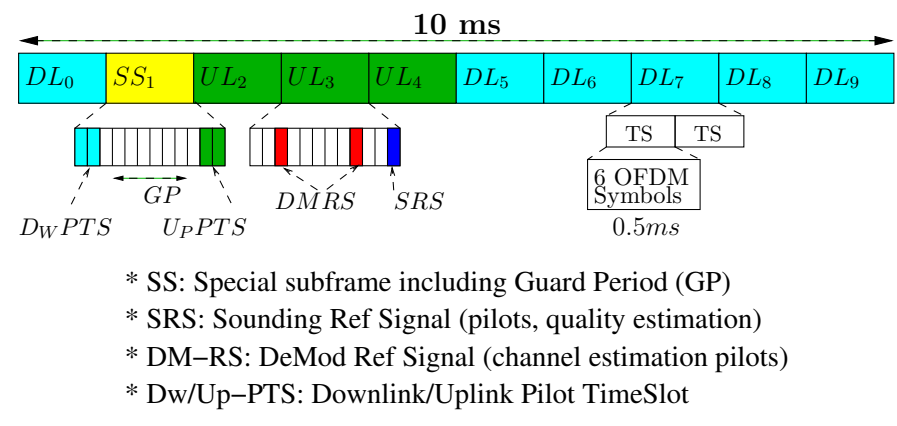

Figure 4: OAI Frame specifications time

Each subframe is divided into 2 time slots. A time slot is consisting of 6 OFDM symbols and a cyclic prefix to avoid inter symbol interference (ISI). The selected OFDM model assumes 512 subcarriers on $5 \mathrm{MHz}$, where 300 subcarriers are actually used. Note that the guard period (GP) in the special subframe is a transition area between the downlink and the uplink part. In the sequel, we intend to exploit this frame structure for the secondary transmissions. Assuming that the secondary system knows the primary frame type, it will modify its frame structure in order to listen the primary frame structure and exploit reference signals (pilots).

Multiple scenarios can be simulated on the OAI platform. The selected calibration algorithm is assessed considering the Rician factor (implying the line of sight (LOS) and non LOS (NLOS)), the number of frames, the SNR, the angle of arrival (AOA), and the transmission power. The pathloss is supposed constant throughout the simulation process. Subsequently, as mentioned before, the synthetic channel model in the simulator is assumed to follow the Ricean model ${ }^{1}$ and is designed through two steps, the first step consists in creating a vector a such:

$\mathbf{a}^{(n)}=\sqrt{\nu} \mathbf{a}^{(n-1)}+\sqrt{(1-\nu)} \sqrt{\left(\frac{k^{\prime}}{2} A\right)} \odot \mathbf{g}+\sqrt{(1-\nu)} \Theta \sqrt{1-k^{\prime}}$

where $k^{\prime}=\frac{1}{1+K}, K$ the linear Ricean factor representing the power ratio $\frac{L O S}{N L O S}, A$ the linear amplitude of taps, $g \sim C \mathcal{N}(0,1), \nu \in[0,1]$ denoted the forgetting factor which adjusts the correlation between the blocks, $n$ indicates recursive steps, $\Theta$ is a vector where first entry is a norm one complex scalar and rest are zeros. Then in the second step, the channel impulse response is designed through this equation:

$$
\begin{gathered}
h(m)=\sum_{l=0}^{N_{p}-1} a[l] \operatorname{sinc}\left(m-F s(l+\beta) \Delta_{\tau_{d}}-\frac{F s}{2} \tau_{\max }\right) \\
\Delta \tau_{d}=\frac{\tau_{\max }}{N_{p}}
\end{gathered}
$$

where $N_{p}$ is the number of path, $\tau_{\max }$ is the maximum delay spread, $F s$ is the sampling frequency, $\beta \in \mathbb{R}$ allows to ensure that the envelope of $h(m)$ is continuous.

The next section addresses the simulation results from OAI.

\section{PERFORMANCE EVALUATING USING OPENAIRINTERFACE}

First of all, we fixed the number of required channel estimation for calibration to $K=10$ ( $K$ channel estimations), in order to perform the simulation. Since, as assessed in our previous study [5], $K \in[10,15]$ allow to find a valuable calibration parameters. Then, assuming that the channel is coherent at least for one frame duration $(10 \mathrm{~ms})$, we store at each side (eNb and UE) one UL/DL channel estimate by frame. Consequently, we need $K$ frames to determine the calibration factors. The $K \mathrm{UL} / \mathrm{DL}$ channels estimates are firstly supposed transmitted and stored perfectly at each side. This first step corresponds to relative calibration observation phase [14].

After a simulation with 100 frames, $K=10$, a Ricean factor equal to $70 d B$, with two power transmissions, $(-5 d B, 5 d B)$ and considering a $2 \times 2$ MIMO channel for the secondary link and a single-input single-output (SISO) channel in primary, the results from simulator shows that without calibration $\mathrm{BF}$ (transmission power $=-5 d B$ ), the primary system transmits correctly, and the secondary transmission is totally perturbed, unlike the $5 d B$ case. It can be explain by

\footnotetext{
${ }^{1}$ The update version of the OAI platform allow to simulate more channel type.
} 
interferences generated by the secondary when we apply a hight power level $(5 d B)$. In this situation, the secondary transmitter prevents a reliable signal transmission from the primary transmitter. Fig. 5 and Fig. 6 illustrate the enhancement, when the BF scheme is applied.

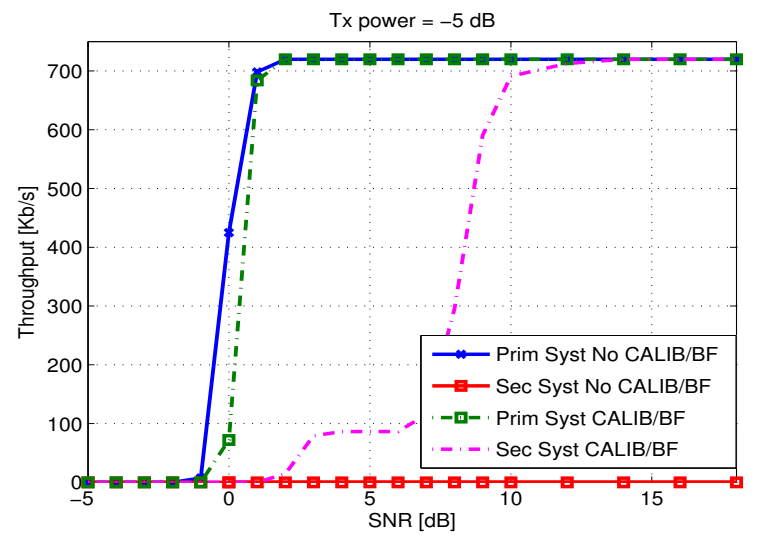

Figure 5: The throughput/SNR for calibration BF and no $\mathrm{BF}$, with a transmission power $=-5 d B$, we observe the null transmission of secondary system, and the improvement of BF.

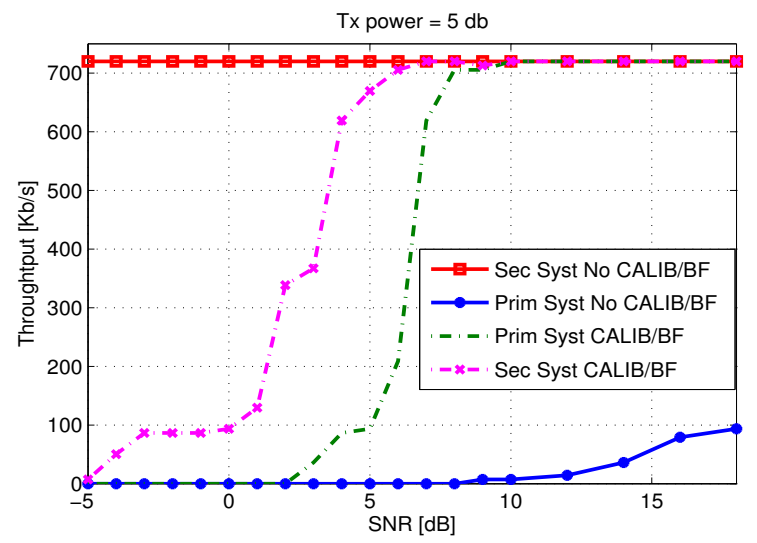

Figure 6: Observation of throughput/SNR for calibration $\mathrm{BF}$ and no $\mathrm{BF}$, with a transmission power $=5 \mathrm{~d} B$, here the secondary transmission totally perturbs the primary system without the beamforming.

In order to evaluate the primary and secondary system coexistence according to the transmit power, we compare the throughput from the primary and the secondary systems. The primary transmission throughput collapses after $5 d B$ due to interference from secondary and lack of interference avoidance techniques (Fig. 7). Nonetheless, in the BF case, all the systems are able to transmit, assuming that the determined calibration parameters are constant during the simulation process (100 frames).

\section{CONCLUSION}

This paper studied the implementation of a reciprocity calibration algorithm in a realistic scenario. The OAI simulator reveals an improvement in terms of throughput, brought

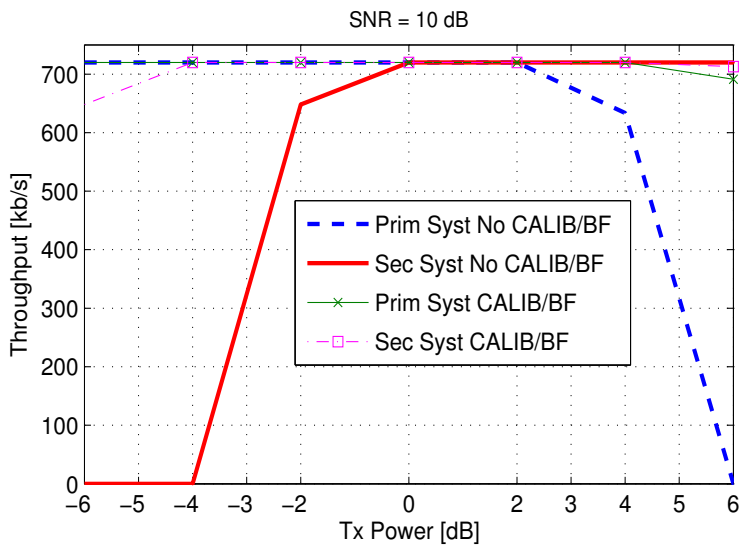

Figure 7: Throughput according to the transmit power and $S N R=10 \mathrm{~dB}$, Rice factor $=70 \mathrm{~dB}$, the primary transmission collapses after $5 d B$ due to interferences

by the selected MIMO calibration method and the beamforming procedure, then it shows a benefit for a power less than $-5 d B$ and greater than $5 d B$ in our specific case. Finally it proves that the calibration is workable, but requires an efficient channel collection technique to avoid the transmission of CSI from the primary to the secondary system, this will be the subject of future works. To conclude, we mention that the channel reciprocity restored through the calibration achievement opens the door to several applications in channel estimation theory, precoding and feedback techniques. To strengthen our conclusions, other algorithms are being tested on the updated version of the OAI taking into account the impact of RF circuitry effects and real channel measurements.

\section{ACKNOWLEDGMENTS}

This collaborative work is supported by the European projects CROWN, ACROPOLIS (advanced coexistence technologies for radio optimisation in licensed and unlicensed spectrum) and SACRA (spectrum and energy efficiency through multi-band cognitive radio).

\section{REFERENCES}

[1] J. Mitola. Cognitive radio: An integrated agent architecture for software defined radio. $\mathrm{PhD}$ thesis, Royal Inst. Tech. (KTH), Stockholm, 2000.

[2] S. Haykin. Cognitive radio: brain-empowered wireless communications. IEEE Journal, Selected Areas in Communications, 23(2):201-220, 2005.

[3] A. Goldsmith, S.A. Jafar, I. Maric, and S. Srinivasa. Breaking spectrum gridlock with cognitive radios: An information theoretic perspective. Proceedings of the IEEE, 97(5):894-914, 2009.

[4] B.D. Van Veen and K.M. Buckley. Beamforming: A versatile approach to spatial filtering. ASSP Magazine, IEEE, 5(2):4-24, 1988.

[5] B. Kouassi, I. Ghauri, B. Zayen, and L. Deneire. On the performance of calibration techniques for cognitive radio systems. In The 14th International Symposium WPMC'11, Brest, France, October 2011. 
[6] N. Mastronardi, P. Lemmerling, and S. Van Huffel. Fast structured total least squares algorithm for solving the basic deconvolution problem. SIAM Journal on Matrix Analysis and Applications, 22:533, 2000.

[7] F. Kaltenberger, H. Jiang, M. Guillaud, and R. Knopp. Relative channel reciprocity calibration in mimo/tdd systems. In Future Network and Mobile Summit, 2010, pages 1-10. IEEE.

[8] I. Markovsky and S. Van Huffel. Overview of total least-squares methods. Signal processing, 87(10):2283-2302, 2007.

[9] The LTE Release website: http://www.3gpp.org/LTE.

[10] Eurecom OpenAirInterface website: http://www.openairinterface.org.

[11] K. Florian, G. Rizwan, K. Raymond, A. Hicham, and B. Christian. Design and implementation of a single-frequency mesh network using openairinterface. EURASIP, 2010.

[12] The CROWN website: http://www.fp7-crown.eu/.

[13] F. Negro, I. Ghauri, and D.T.M. Slock. Transmission techniques and channel estimation for Spatial Interweave TDD Cognitive Radio systems. In 43rd Asilomar Conference on Signals, Systems and Computers, pages 523-527. IEEE, 2010.

[14] M. Guillaud, D.T.M. Slock, and R. Knopp. A practical method for wireless channel reciprocity exploitation through relative calibration. 8th ISSPA 2005, Sydney, Australia, pages 403-406, Aug. 29-Sep. 1,2005. 